\title{
Características del registro de historias clínicas en un hospital al sur del Perú
}

\author{
Cender Udai Quispe-Juli ${ }^{1, a}$, Rossi Yessenia Navarro-Navides ${ }^{1}$, Luis Gustavo Velásquez-Chahuares $^{1, a}$, Frank Pinto-Martínez ${ }^{1}$, \\ Elvis Olivares-Alegría ${ }^{1}$
}

\section{RESUMEN}

Objetivo: determinar las características de registro de las historias clínicas de hospitalización en el Hospital III Yanahuara de la ciudad de Arequipa, Perú.

Material y métodos: El estudio fue de tipo observacional, transversal y retrospectivo. Se evaluaron 225 historias clínicas de hospitalización en el mes de noviembre del 2015. Se utilizó una ficha que consta de 15 ítems; cada ítem se evaluó mediante una escala en: “muy mal”, “mal”, “aceptable”, "bien” y “muy bien”. Se hizo un análisis descriptivo a través del cálculo de frecuencias.

Resultados: Los ítems con mayor proporción de datos de registro aceptable fueron: indicación terapéutica clara (84\%), evolución (74,7\%), juicio clínico (70,7\%), indicación terapéutica completa y ordenada (54,2\%), enfermedad actual (50,2\%), y exploración física $(43,1 \%)$. Los ítems muy bien registrados fueron: indicación de pruebas y procedimientos (97,3\%), identificación de médico $(91,1 \%)$ y alergias $(67,1 \%)$. Los ítems muy mal registrados fueron: motivo de ingreso $(91,1 \%)$, hábitos vitales $(72,9 \%)$ y tratamiento previo $(38,2 \%)$.

Conclusiones: la mayoría de las historias clínicas de hospitalización se caracteriza por un registro aceptable de la mayoría de ítems evaluados; sin embargo presentan deficiencias notables en algunos ítems.

Palabras clave: registros médicos, control de calidad, hospitalización.

\section{Characteristics of registration of medical records in a hospital in southern Peru}

\section{ABSTRACT}

Objective: To determine the characteristics of registration of medical records of hospitalization in the Hospital III Yanahuara in Arequipa, Peru.

Material and methods: The study was observational, cross-sectional and retrospective. 225 medical records of hospitalization were evaluated in November 2015. A tab consisting of 15 items was used; each item was assessed using a scale: "very bad", "bad", "acceptable", "good" and "very good". A descriptive analysis was done by calculating frequency.

Results: Items with a higher proportion of acceptable registration data were: clear therapeutic indication (84\%), clinical evolution (74.7\%), diagnosis (70.7\%), complete and orderly therapeutic indication (54.2\%), medical history taking (50.2\%) and physical examination (43.1\%). The very well recorded items were: indication of tests and procedures (97.3\%), medical identification (91.1\%) and allergies (67.1\%). Very bad recorded items were: reason for admission (91.1\%), life habits (72.9\%) and prior treatment (38.2\%).

Conclusions: Most medical records of hospitalization are characterized by an acceptable record of most evaluated items; however they have notable deficiencies in some items.

Key words: medical records, quality control, hospitalization.

1. Facultad de Medicina Humana, Universidad Nacional de San Agustín. Arequipa, Perú

a. Sociedad Científica de Estudiantes de Medicina Agustinos (SOCIEMA). 


\section{INTRODUCCIÓN}

La historia clínica es un documento esencial para la asistencia médica y sanitaria, indispensable para el diagnóstico y seguimiento de los pacientes, fundamental para orientar el tratamiento; que tiene implicancias legales y desempeña una función importante en la investigación, docencia, evaluación y mejoramiento de la calidad y gestión de recursos ${ }^{(1,2)}$. Es conocido que el estudio del registro o llenado de historias clínicas es un instrumento para la mejora de estándares de atención ${ }^{(2)}$; que tiene incluso impacto económico especialmente en instituciones de salud privadas $^{(3,4)}$. Los procesos de retroalimentación tienen efectos favorables: mejorando la práctica profesional y evidenciando un mejor desempeño. Por tanto, un mal registro en la historia clínica disminuiría la calidad de atención aumentando la morbimortalidad de la población ${ }^{(3)}$.

Se han realizado algunos estudios en nuestro país (Perú), en diferentes establecimientos de salud y contextos socioculturales, en los que se encontró resultados heterogéneos en el registro de historia clínica, donde la mayoría de ellos tenía una deficiente calidad de registro de datos ${ }^{(2,4,5,6)}$. No hay estudios realizados en el contexto local (Arequipa), que permitan plantear intervenciones pertinentes para la mejora de la atención en salud. El objetivo del presente estudio fue determinar las características de registro en las historias clínicas de hospitalización del Hospital III Yanahuara de la ciudad de Arequipa, Perú.

\section{MATERIALES Y MÉTODOS}

El estudio fue de tipo observacional, transversal y retrospectivo. El ámbito de investigación fue el Hospital III Yanahuara del Seguro Social de Salud, ubicado en la ciudad de Arequipa, Perú; durante el mes noviembre del 2015. Se evaluaron las historias clínicas del servicio de hospitalización. Se incluyeron las historias clínicas de los servicios de medicina interna, ginecología-obstetricia y cirugía que pertenecían a pacientes recientemente dados de alta (un mes de antigüedad). Se excluyeron las historias incompletas (que le falta una o varias hojas), historias deterioradas. Se realizó un muestreo no probabilístico, tipo censal, de 230 historias clínicas.

Se usó una ficha para la recolección de datos; evaluable para cada ítem mediante una escala en: "muy mal", "mal", "aceptable", "bien” y "muy bien"; utilizado en un estudio previo en España ${ }^{(1)}$, que fue adaptado, según la Norma Técnica de la Historia Clínica de los Establecimientos del Sector Salud elaborada por el Ministerio de Salud de Perú ${ }^{(7)}$. El mismo que consta de 15 ítems, los ítems y los criterios de calificación para cada uno se especifica en la Tabla 1. La adaptación se realizó para tres ítems: identificación del médico, datos generales, indicación terapéutica completa y ordenada. Los criterios de evaluación (de los ítems modificados) no se aplicaban al contexto local, ya que tenían una mayor exigencia, según la norma técnica ${ }^{(7)}$. Únicamente se hizo una validación lógica o aparente, a juicio de expertos. Cada ficha fue llenada según los criterios establecidos; previa evaluación y discusión de todos los investigadores; esto último con el fin de controlar el sesgo interobservador.

El estudio se realizó con aceptación de la administración del hospital, a la que se entregó una copia del informe final. Los datos fueron manejados con discreción: se respetó el anonimato de los pacientes, al no incluir en la base de datos el nombre ni ningún dato que pueda identificarlos. Además únicamente los investigadores responsables tuvieron acceso a los mismos.

La información se procesó con el paquete Microsoft Excel 2010 y se hizo un análisis descriptivo a través del cálculo de frecuencias. 
Tabla 1: Contenido de la ficha de evaluación

\begin{tabular}{|c|c|c|c|c|c|}
\hline & $\begin{array}{l}\text { Muy } \\
\text { mal }\end{array}$ & Mal & Aceptable & Bien & Muy bien \\
\hline Legibilidad & $\begin{array}{l}\text { Todo } \\
\text { ilegible }\end{array}$ & $\begin{array}{l}\text { Muchos contenidos } \\
\text { ilegibles }\end{array}$ & $\begin{array}{l}\text { Alguna palabra } \\
\text { difícil }\end{array}$ & $\begin{array}{l}\text { Lectura de } \\
\text { corrido }\end{array}$ & $\begin{array}{l}\text { Lectura de } \\
\text { corrido } \\
\text { muy rápida } \\
\text { para } \\
\text { cualquiera }\end{array}$ \\
\hline $\begin{array}{l}\text { Identificación } \\
\text { de médico }\end{array}$ & $\begin{array}{l}\text { No } \\
\text { figura } \\
\text { o solo } \\
\text { está la } \\
\text { firma }\end{array}$ & & $\begin{array}{l}\text { Firma, nombres y } \\
\text { apellidos }\end{array}$ & & $\begin{array}{l}\text { Firma, } \\
\text { nombres y } \\
\text { apellidos, } \\
\text { colegiatura }\end{array}$ \\
\hline $\begin{array}{l}\text { Datos } \\
\text { generales }\end{array}$ & $\begin{array}{l}\text { No } \\
\text { constan }\end{array}$ & $\begin{array}{l}\text { Solo está apellidos y } \\
\text { nombres, documento } \\
\text { nacional de } \\
\text { identidad }\end{array}$ & $\begin{array}{l}\text { Sexo, edad, fecha y } \\
\text { lugar de nacimiento, } \\
\text { procedencia además } \\
\text { de lo anterior }\end{array}$ & $\begin{array}{l}\text { Grado de } \\
\text { instrucción, } \\
\text { estado } \\
\text { civil, } \\
\text { ocupación } \\
\text { u oficio } \\
\text { además de } \\
\text { lo anterior }\end{array}$ & $\begin{array}{l}\text { Grupo } \\
\text { sanguíneo y } \\
\text { factor Rh, } \\
\text { teléfono y } \\
\text { nombre de } \\
\text { familiar de } \\
\text { contacto } \\
\text { además de } \\
\text { lo anterior }\end{array}$ \\
\hline $\begin{array}{l}\text { Motivo de } \\
\text { ingreso }\end{array}$ & $\begin{array}{l}\text { No } \\
\text { consta }\end{array}$ & & & & Consta \\
\hline $\begin{array}{l}\text { Antecedentes } \\
\text { personales }\end{array}$ & $\begin{array}{l}\text { No } \\
\text { constan }\end{array}$ & $\begin{array}{l}\text { Médicos y } \\
\text { quirúrgicos sin } \\
\text { informar de } \\
\text { hipertensión, } \\
\text { diabetes ni } \\
\text { dislipidemia }\end{array}$ & $\begin{array}{l}\text { Médicos y } \\
\text { quirúrgicos } \\
\text { incluyendo los } \\
\text { anteriores }\end{array}$ & $\begin{array}{l}\text { Médicos y } \\
\text { quirúrgicos } \\
\text { con algunas } \\
\text { fechas }\end{array}$ & $\begin{array}{l}\text { Médicos y } \\
\text { quirúrgicos } \\
\text { con fechas } \\
\text { del } \\
\text { padecimiento } \\
\text { e } \\
\text { información } \\
\text { sobre } \\
\text { evaluaciones }\end{array}$ \\
\hline $\begin{array}{l}\text { Hábitos } \\
\text { vitales } \\
\text { (alcohol, } \\
\text { tabaco, } \\
\text { dieta y } \\
\text { actividad } \\
\text { física) }\end{array}$ & $\begin{array}{l}\text { No } \\
\text { constan }\end{array}$ & $\begin{array}{l}\text { Consta solo } 1 \text { de: } \\
\text { alcohol, tabaco, } \\
\text { dieta y actividad } \\
\text { física }\end{array}$ & $\begin{array}{l}\text { Consta } 2 \text { de: alcohol, } \\
\text { tabaco, dieta y } \\
\text { actividad física }\end{array}$ & $\begin{array}{l}\text { Consta } 3 \text { o } \\
\text { más de: } \\
\text { alcohol, } \\
\text { tabaco, } \\
\text { dieta y } \\
\text { actividad } \\
\text { física }\end{array}$ & \\
\hline Alergias & $\begin{array}{l}\text { No } \\
\text { constan }\end{array}$ & & & & Consta \\
\hline $\begin{array}{l}\text { Tratamiento } \\
\text { previo }\end{array}$ & $\begin{array}{l}\text { No } \\
\text { consta }\end{array}$ & Manera genérica & Algunos & $\begin{array}{l}\text { Todos con } \\
\text { algunas } \\
\text { dosis }\end{array}$ & $\begin{array}{l}\text { Todos con } \\
\text { todas las } \\
\text { dosis }\end{array}$ \\
\hline $\begin{array}{l}\text { Enfermedad } \\
\text { actual } \\
\text { (anamnesis) }\end{array}$ & $\begin{array}{l}\text { No } \\
\text { consta }\end{array}$ & $\begin{array}{l}\text { Insuficiente para } \\
\text { establecer juicio } \\
\text { clínico }\end{array}$ & $\begin{array}{l}\text { Aceptable pero } \\
\text { faltan datos }\end{array}$ & $\begin{array}{l}\text { Aceptable- } \\
\text { mente } \\
\text { completa }\end{array}$ & $\begin{array}{l}\text { Completa y } \\
\text { minuciosa }\end{array}$ \\
\hline $\begin{array}{l}\text { Exploración } \\
\text { física }\end{array}$ & $\begin{array}{l}\text { No } \\
\text { figura }\end{array}$ & Breve e incompleta & $\begin{array}{l}\text { Incluye aspectos } \\
\text { claves pero de forma } \\
\text { incompleta }\end{array}$ & $\begin{array}{l}\text { Tiene } \\
\text { aspectos } \\
\text { claves y es } \\
\text { completa }\end{array}$ & $\begin{array}{l}\text { Completa y } \\
\text { minuciosa }\end{array}$ \\
\hline
\end{tabular}




\begin{tabular}{|c|c|c|c|c|c|}
\hline $\begin{array}{l}\text { Juicio clínico } \\
\text { (diagnósticos } \\
\text { presuntivos y } \\
\text { definitivos) }\end{array}$ & $\begin{array}{l}\text { No } \\
\text { figura }\end{array}$ & Está incompleto & $\begin{array}{l}\text { Completo pero sólo } \\
\text { referido a un } \\
\text { proceso }\end{array}$ & $\begin{array}{l}\text { Informa } \\
\text { sobre todas } \\
\text { la } \\
\text { enfermeda } \\
\text { des del } \\
\text { paciente }\end{array}$ & $\begin{array}{l}\text { Incluye } \\
\text { además } \\
\text { datos de } \\
\text { diagnóstico } \\
\text { diferencial } \\
\text { entre ellos }\end{array}$ \\
\hline $\begin{array}{l}\text { Indicación de } \\
\text { pruebas o } \\
\text { procedimien- } \\
\text { tos }\end{array}$ & $\begin{array}{l}\text { No } \\
\text { constan }\end{array}$ & & & & consta \\
\hline $\begin{array}{l}\text { Evolución (la } \\
\text { evolución de } \\
\text { los síntomas } \\
\text { o los signos, } \\
\text { resultado de } \\
\text { las pruebas, } \\
\text { juicio clínico } \\
\text { actualizado y } \\
\text { la actitud a } \\
\text { tomar) }\end{array}$ & $\begin{array}{l}\text { No } \\
\text { consta }\end{array}$ & & $\begin{array}{l}\text { Consta } 1 \text {-2 de: } \\
\text { evolución de los } \\
\text { síntomas o signos; } \\
\text { resultado de las } \\
\text { pruebas; juicio } \\
\text { clínico actualizado y } \\
\text { la actitud a tomar }\end{array}$ & & $\begin{array}{l}\text { Consta 3-4 } \\
\text { de: } \\
\text { evolución } \\
\text { de los } \\
\text { síntomas o } \\
\text { signos; } \\
\text { resultado } \\
\text { de las } \\
\text { pruebas; } \\
\text { juicio } \\
\text { clínico } \\
\text { actualizado } \\
\text { y la actitud } \\
\text { a tomar }\end{array}$ \\
\hline $\begin{array}{l}\text { Indicación } \\
\text { terapéutica } \\
\text { completa y } \\
\text { ordenada } \\
\text { (medicamento, } \\
\text { concentración, } \\
\text { presentación, } \\
\text { dosis, vía, } \\
\text { frecuencia y } \\
\text { duración) }\end{array}$ & $\begin{array}{l}\text { Incom } \\
\text { pleta y } \\
\text { desord } \\
\text { enada } \\
\text { para } \\
\text { todos } \\
\text { los } \\
\text { medica } \\
\text { mentos }\end{array}$ & $\begin{array}{l}\text { Incompleta y } \\
\text { desordenada para la } \\
\text { mayoría de } \\
\text { medicamentos }\end{array}$ & $\begin{array}{l}\text { Completa (puede no } \\
\text { precisar: } \\
\text { presentación, } \\
\text { duración) pero } \\
\text { desordenada para la } \\
\text { mayoría de } \\
\text { medicamentos }\end{array}$ & $\begin{array}{l}\text { Completa y } \\
\text { ordenada } \\
\text { para la } \\
\text { mayoría de } \\
\text { medicamen } \\
\text { tos }\end{array}$ & $\begin{array}{l}\text { Completa y } \\
\text { ordenada } \\
\text { (medicamen } \\
\text { to, } \\
\text { concentra- } \\
\text { ción, } \\
\text { presentación } \\
\text { dosis, } \\
\text { vía, } \\
\text { frecuencia } \\
\text { y duración) } \\
\text { para todos } \\
\text { los } \\
\text { medicamentos }\end{array}$ \\
\hline $\begin{array}{l}\text { Indicación } \\
\text { terapéutica } \\
\text { clara }\end{array}$ & Confusa & & $\begin{array}{l}\text { Aceptablemente } \\
\text { clara }\end{array}$ & & Muy clara \\
\hline
\end{tabular}

\section{RESULTADOS}

Se identificaron 230 historias clínicas; de las cuales 225 fueron evaluables, se excluyeron cuatro por estar incompletas y una por estar deteriorada. Las frecuencias por ítem evaluado mostró que la mayor proporción de datos con registro aceptable fueron: indicación terapéutica clara, evolución, juicio clínico, indicación terapéutica completa y ordenada, enfermedad actual, y exploración física (Tabla 2). 
Tabla 2. Frecuencias de las características de registro para cada ítem valorado

\begin{tabular}{|c|c|c|c|c|c|c|c|c|c|c|}
\hline \multirow[t]{2}{*}{ Ítem Valorado } & \multicolumn{2}{|c|}{ Muy Mal } & \multicolumn{2}{|c|}{ Mal } & \multicolumn{2}{|c|}{ Aceptable } & \multicolumn{2}{|c|}{ Bien } & \multicolumn{2}{|c|}{ Muy Bien } \\
\hline & $\mathbf{n}$ & (\%) & $n$ & (\%) & $\mathbf{n}$ & (\%) & $\mathrm{n}$ & (\%) & $n$ & (\%) \\
\hline Legibilidad & 6 & $(2,7)$ & 105 & $(46,7)$ & 76 & $(33,8)$ & 36 & (16) & 2 & $(0.9)$ \\
\hline $\begin{array}{l}\text { Identificación de } \\
\text { médico }\end{array}$ & 15 & $(6,7)$ & & & 5 & $(2,2)$ & & & 205 & $(91,1)$ \\
\hline Datos generales & 1 & $(0,4)$ & 5 & $(2,2)$ & 65 & $(28,9)$ & 133 & $(59,1)$ & 21 & $(9,3)$ \\
\hline Motivo de ingreso & 205 & $(91,1)$ & & & & & & & 20 & (8.9) \\
\hline $\begin{array}{l}\text { Antecedentes } \\
\text { personales }\end{array}$ & 8 & $(3,6)$ & 35 & $(15,6)$ & 80 & $(35,6)$ & 96 & $(42,7)$ & 6 & $(2,7)$ \\
\hline Hábitos vitales & 164 & $(72,9)$ & 12 & $(5,3)$ & 21 & $(9,3)$ & 27 & (12) & & \\
\hline Alergias & 74 & $(32,9)$ & & & & & & & 151 & $(67,1)$ \\
\hline $\begin{array}{l}\text { Tratamiento } \\
\text { previo }\end{array}$ & 86 & $(38,2)$ & 41 & $(18,2)$ & 39 & $(17,3)$ & 54 & (24) & 5 & $(2,2)$ \\
\hline $\begin{array}{l}\text { Enfermedad } \\
\text { actual }\end{array}$ & 10 & $(4,4)$ & 64 & $(28,4)$ & 113 & $(50,2)$ & 35 & $(15,6)$ & 3 & $(1,3)$ \\
\hline Exploración física & 11 & $(4,9)$ & 92 & $(40,9)$ & 97 & $(43,1)$ & 24 & $(10,7)$ & 1 & $(0,4)$ \\
\hline Juicio clínico & 3 & $(1,3)$ & 39 & $(17,3)$ & 159 & $(70,7)$ & 22 & $(9,8)$ & 2 & $(0,9)$ \\
\hline $\begin{array}{l}\text { Indicación de } \\
\text { pruebas o } \\
\text { procedimientos }\end{array}$ & 6 & $(2,7)$ & & & & & & & 219 & $(97,3)$ \\
\hline Evolución & 1 & $(0,4)$ & & & 168 & $(74,7)$ & & & 56 & $(24,9)$ \\
\hline $\begin{array}{l}\text { Indicación } \\
\text { terapéutica } \\
\text { completa y } \\
\text { ordenada }\end{array}$ & 2 & $(0,9)$ & 23 & $(10,2)$ & 122 & $(54,2)$ & 77 & $(34,2)$ & 1 & $(0,4)$ \\
\hline $\begin{array}{l}\text { Indicación } \\
\text { terapéutica clara }\end{array}$ & 31 & $(13,8)$ & & & 189 & (84) & & & 5 & $(2,2)$ \\
\hline
\end{tabular}

Los ítems que presentaron principalmente datos muy bien registrados fueron: indicación de pruebas y procedimientos, identificación de médico y alergias. Los ítems con muy mal registro fueron: motivo de ingreso, hábitos vitales y tratamiento previo.

\section{DISCUSIÓN}

Un número importante de historias clínicas $(46,7 \%)$ presentaban una mala legibilidad (muchos contenidos ilegibles), mayor a lo encontrado en el Callao $(31,9 \%)^{(5)}$ y a lo reportado en España $(4,5 \%)^{(1)}$; en relación a la indicación terapéutica clara se encontró un registro confuso en el 13,8\%, notablemente mayor a España $(0,9 \%)^{(1)}$; esto se debería a una "peor letra" de los médicos con respecto a la población, el poco tiempo para llevarlo a cabo e incluso la falta de interés ${ }^{(8)}$. Con respecto a la identificación del médico, se halló que un 91,1\% registró muy bien sus datos (firma, nombres y apellidos, número de colegiatura); similar al encontrado en Lima $(97,2 \%){ }^{(4)}$; pero mayor a los obtenidos otros hospitales del país ${ }^{(3)}$. Los datos generales estaban bien registrados o de forma aceptable en la mayoría de las historias, similar lo hallado en Chiclayo ${ }^{(2)}$, probablemente estos datos sean recogidos ampliamente como consecuencia del formato pre-establecido del hospital. El motivo de ingreso tenía un muy mal registro (no constaba) en más del $90 \%$; esto podría explicarse por la ausencia del ítem en el formato de la historia clínica de hospitalización, posiblemente porque no está contemplado en la norma técnica ${ }^{(7)}$, por lo que se omite su registro.

En cuanto a los antecedentes personales, más del $77 \%$ presentó un registro por lo menos aceptable; mayor a lo encontrado en el Callao $(38,5 \%)^{(5)}$ pero similar a lo reportado en España $(76 \%)^{(2)}$, otros estudios reportan resultados inferiores $(13,4 \%){ }^{(10)}$; existen escasos estudios sobre la calidad de los antecedentes recogidos en historias clínicas ${ }^{(9)}$. Los hábitos vitales, entendidos como estilos de vida, no constaban en la mayoría de historias (72,3\%); consideramos que esta 
información es muy importante para el diagnóstico, tratamiento e incluso pronóstico de múltiples enfermedades; cuyo registro no debe limitarse a: dieta, actividad física, consumo de tabaco y alcohol sino extenderse en lo relacionado a sueño y descanso adecuados, bienestar emocional y mental, entre otros ${ }^{(10)}$. Llama la atención que a pesar de las potenciales consecuencias médico-legales haya una gran ausencia de registro de alergias (32,9\%); superior a lo reportado en España $(20,7 \%)^{(1)}$; sin embargo menor a lo encontrado en otro estudio en el mismo país (ausencia del registro médico de $50 \%$ de alergias farmacológicas y $100 \%$ de alergias no farmacológicas), éste último realizado a través de aplicaciones informáticas, donde se encontró una variabilidad importante en el registro de alergias por profesionales sanitarios ${ }^{(11)}$; lo que también explicaría ésta heterogeneidad de resultados. Existe una ausencia considerable de registro de los tratamientos previos $(38,2 \%)$, se le debería dar más importancia a esta información para un mejor manejo terapéutico (evitar posibles interacciones farmacológicas) y un mejor control de reacciones adversas. En lo que respecta a la enfermedad actual (anamnesis), se encontró que la mitad de las historias presentan un registro aceptable, sin embargo un porcentaje considerable $(32,9 \%)$ presentó un mal o muy mal registro; esto podría provocar un retraso o error en el diagnóstico y tratamiento, una mayor estancia hospitalaria, y en el peor de los casos, complicaciones que pondrían en riesgo al paciente ${ }^{(6)}$.

El registro de la exploración física fue aceptable (incluye aspectos clave pero de forma incompleta) en menos del 50\%; en 11 historias clínicas no figuraba registro alguno y solo una lo realizó de forma completa y minuciosa. Otros estudios evalúan únicamente la presencia o ausencia del mismo, mas no las características de su registro ${ }^{(3,5)}$. En tres historias clínicas $(1,3 \%)$ no figuraba ni el diagnóstico presuntivo ni el definitivo (juicio clínico); otros estudios mostraron también que menos del $10 \%$ no registraban este ítem ${ }^{(1,2,4,5)}$, sin embargo resulta preocupante dado que esto es imprescindible para un adecuado manejo terapéutico. Respecto al plan de trabajo, no constaba en $2,7 \%$ de historias; menor a lo encontrado en otros trabajos previos, aproximadamente del $20 \%^{(3,4)}$. En la mayoría de historias (75\%), el ítem de evolución presentó deficiencias en el llenado de las mismas, lo cual probablemente se debió a la falta de exigencia o interés. La indicación terapéutica era completa (especificaba: medicamento, concentración, presentación, dosis, vía, frecuencia y duración) y ordenada para todos los medicamentos, solo en una historia; estos resultados difieren ampliamente con lo obtenido en España $(94,6 \%)^{(1)}$; otros estudios no son comparables debido a los diferentes criterios de análisis utilizados para la categorización de este ítem.

Finalmente, la toma correcta de decisiones médicas depende en gran medida de la información disponible en historia clínica, por lo que resultan preocupantes algunos hallazgos. Esta situación puede deberse al error médico originado por la fatiga asociada a la sobrecarga laboral, por exceso de horas de trabajo, causada por la presión generada de un sistema de salud que no satisface la demanda de atención sanitara de la población o por aspiraciones económicas personales ${ }^{(12)}$.

Podemos considerar limitaciones del estudio el muestreo por conveniencia, el que los datos se refieran a un solo hospital y se limite a un periodo de tiempo breve; por ello su reproducibilidad externa es discutible; también hubiera sido útil conocer las características evaluadas de acuerdo a los diferentes servicios de hospitalización, por lo que recomendamos estos aspectos sean tomados en cuenta en futuras investigaciones. Es necesario que se realicen más estudios en diferentes hospitales de los diferentes sectores de salud (Ministerio de salud, Fuerzas Armadas, Sanidad de la Policía Nacional, clínicas privadas) para conocer la verdadera magnitud del problema. Este estudio nos permite hacer una aproximación a la realidad asistencial sanitaria del Perú.

En conclusión, la mayoría de las historias clínicas de hospitalización se caracteriza por un registro aceptable de la mayoría de ítems evaluados, sin embargo presentan un mal o muy mal registro en algunos aspectos: motivo de ingreso, hábitos vitales y tratamiento previo.

Agradecimiento: A los médicos, Idelfonso Lira Linares, María L. Viza Butrón por los aportes y sugerencias; a los miembros administrativos del Hospital III Yanahuara, por las facilidades prestadas para la realización del estudio.

\section{REFERENCIAS BIBLIOGRÁFICAS}

1. Wikman P, Safont P, Merino J, Martínez-Baltanás A, Matarranz del Amo M, López Calleja E. Intervención para la mejora de la calidad de las historias clínicas en un Servicio de Medicina Interna. Rev Clín Esp. 2009;209(8):391-395.

2. Puescas-Sánchez PR, Diaz-Nolazco MA, Diaz-Velez C. Calidad técnica de las historias clínicas de los pacientes hospitalizados 
en un hospital EsSalud de Chiclayo 2008-2010. Rev Cuerpo Méd Hosp Nac Alman Agui Asen. 2012;5(2):5-10.

3. Llanos-Zavalaga LF, Mayca-Pérez J, Chumbes-Navarro GC. Auditoría médica de historias clínicas en consulta externa de cuatro hospitales públicos peruanos. Rev Med Hered. 2006;17(4):220-226.

4. Matzumura-Kasano JP, Gutiérrez-Crespo H, Sotomayor-Salas J, Pajuelo-Carrasco G. Evaluación de la calidad de registro de historias clínicas en consultorios externos del servicio de medicina interna de la Clínica Centenario Peruano Japonesa, 2010-2011. An Fac Med. 2014;75(3):251-257.

5. Zafra-Tanaka J, Veramendi-Espinoza L, Villa-Santiago $\mathrm{N}$, Zapata-Sequeiros M, Yovera-Leyva E, Urbina-Yale G, et al. Calidad de registro en historias clinicas en un centro de salud del Callao, Peru 2013. Carta al Editor. Rev Peru Med Exp Salud Pública. 2013;30(4):719-720.

6. Bocanegra-Garcia SM, Bocanegra-Garcia GE, Alvarado-Caceres VM. Nivel de calidad del registro de las historias clínicas de pacientes apendicectomizados en el Hospital Belén de Trujillo de enero a junio de 2007. Rev Med Vallej. 2007;5(2):115-124.

7. Ministerio de Salud del Perú. Norma técnica de la historia clínica de los establecimientos del sector salud. N.T. N ${ }^{\circ} 022-$ MINSA/DGSP-V.02. Lima: Dirección general de salud de las personas; 2005.

8. Robaina-Bordon JM, Morales-Castellano E, Lopez-Rodeiguez JF, Sosa-Enriquez M. La letra de médico. Rev Osteoporos Metab Miner. 2014;6(4):122-126.
9. Expósito-Tirado JA, Vergara-Diaz G, Guerrero-Vázquez R, Vázquez-González A, Márquez-Galán JL, Garnacho-Montero J, et al. Calidad de los antecedentes personales en la historia clínica electrónica hospitalaria. Carta al editor. Rev Calid Asist. 2014;29(6):362-365.

10. Mora-Ripoll R. Medicina del estilo de vida: la importancia de considerar todas las causas de la enfermedad. Rev Psiquiatr y Salud Ment. 2012;5(1):48-52.

11. Caro JM, Jiménez MJ, Escribano I, Ferrari JM. Variabilidad en el registro de alergias por profesionales sanitarios en un hospital de tercer nivel. Pharm Care esp. 2015;17(6):732-744.

12. Tejeda-Mariaca JE, Pizango-Mallqui O, Albuquerque-Duglio MA. Error médico asociado a múltiples trabajos: Un problema con trasfondo económico. Carta al editor. CIMEL. 2014;28(2):96.

Fuentes de financiamiento:

Este artículo ha sido financiado por los autores.

Conflictos de interés:

Los autores declaran no tener ningún conflicto de interés.

\section{Correspondencia:}

Cender Udai Quispe-Juli

Dirección: Urb. Las Orquídeas K-20, Cercado, Arequipa, Perú.

Teléfono: (51) 950853190

Correo electrónico: cenderqj@gmaill.com

Recibido: 24 de julio de 2016

Aprobado: 06 de setiembre de 2016 